\title{
BMJ Open Exploring digital health interventions for pregnant women at high risk for pre- eclampsia and eclampsia in low-income and-middle-income countries: a scoping review
}

\author{
Anam Shahil Feroz (D) ,, ${ }^{1,2}$ Noreen Afzal (D) ," Emily Seto ${ }^{2,4}$
}

To cite: Shahil Feroz A, Afzal N, Seto E. Exploring digital health interventions for pregnant women at high risk for pre-eclampsia and eclampsia in low-income and-middle-income countries: a scoping review. BMJ Open 2022;12:e056130. doi:10.1136/ bmjopen-2021-056130

- Prepublication history and additional supplemental material for this paper are available online. To view these files, please visit the journal online (http://dx.doi.org/10.1136/ bmjopen-2021-056130).

Received 04 August 2021 Accepted 07 January 2022

Check for updates

(C) Author(s) (or their employer(s)) 2022. Re-use permitted under CC BY-NC. No commercial re-use. See rights and permissions. Published by BMJ.

${ }^{1}$ Community Health Sciences, Aga Khan University, Karachi, Pakistan

${ }^{2}$ Institute of Health Policy, Management and Evaluation, University of Toronto, Toronto, Ontario, Canada

${ }^{3}$ Dean's Office, The Aga Khan University Medical College Pakistan, Karachi, Sindh,

Pakistan

${ }^{4}$ Centre for Global eHealth Innovation, University Health Network, Toronto, Ontario, Canada

Correspondence to

Dr Anam Shahil Feroz;

anam.shahil@mail.utoronto.ca

\section{ABSTRACT}

Objective To explore digital health interventions that have been used to support pregnant women at high risk for pre-eclampsia/eclampsia (HRPE/E) in low-income and middle-income countries (LMICs).

Design Scoping review.

Data source EMBASE, MEDLINE, Cochrane Central Register of Controlled Trials, Cochrane Database of Systematic Reviews and CINAHL were searched between 1 January 2000 and 20 0ctober 2020.

Eligibility criteria The review included original research studies that were published in English, involved pregnant women at HRPE/E and implemented digital health interventions for PE/E in LMICs.

Data extraction and synthesis Two reviewers independently completed the data extraction for each of the 19 final articles. An inductive approach was used to thematically organise and summarise the results from the included articles.

Results A total of 19 publications describing 7 unique studies and 9 different digital health interventions were included. Most studies were conducted in South Asia and sub-Saharan Africa $(n=16)$. Of nine unique digital health interventions, two served the purpose of predicting risk for adverse maternal health outcomes while seven focused on monitoring high-risk pregnant women for PE/E. Both of these purposes used mobile phone applications as interface to facilitate data collection, decision making, and communication between health workers and pregnant women. The review identified key functions of interventions including data collection, prediction of adverse maternal outcomes, integrated diagnostic and clinical decision support, and personal health tracking. The review reported three major outcomes: maternal health outcomes including maternal and neonatal morbidity and mortality $(n=4)$; usability and acceptability including easeof-use, and perceived usefulness, $(n=5)$; and intervention feasibility and fidelity including accuracy of device, and intervention implementation $(n=7)$.

Conclusion Although the current evidence base shows some potential for the use of digital health interventions for $\mathrm{PE} / \mathrm{E}$, more prospective experimental and longitudinal studies are needed prior to recommending the use of digital health interventions for PE/E.
Strengths and limitations of this study

- First scoping review to explore the use of digital health interventions (DHIs) in low-income and middle-income countries (LMICs) to support pregnant women at high risk for pre-eclampsia/eclampsia (PE/E).

- This scoping review has identified several gaps in the area of DHIs use for PE/E in LMICs which can be explored through future research.

- The high heterogeneity of the DHls and study outcomes limited the interpretation of the studies through quantitative analysis.

- This review only included peer-reviewed articles and papers published in the English language.

- The review did not include information that may have been found in other databases and sources (abstracts, reviews, conference proceedings, opinion papers, books).

\section{INTRODUCTION}

Approximately $16 \%$ of all maternal deaths in low-income and middle-income countries (LMICs) are attributable to pre-eclampsia/ eclampsia $(\mathrm{PE} / \mathrm{E}) .^{1}$ High maternal mortality from PE/E results from: (1) lack of early identification and treatment of pregnant women, (2) difficulties in reaching treatment centres and (3) poor health-seeking behaviours linked with low patient education. $^{2}$ To meet the United Nations Sustainable Developmental Goal target 3.1 of reducing the maternal mortality ratio to less than 70/100 000 live births by 2030 , innovations are required to decrease $\mathrm{PE} / \mathrm{E}$-related mortality. ${ }^{3}$

The most effective strategies to ensure early diagnosis and management of $\mathrm{PE} / \mathrm{E}$ include self-monitoring of blood pressure, use of magnesium sulfate therapy, proteinuria determinations and timely delivery. ${ }^{1}$ 
International guidelines including the European Society of Hypertension, American Heart Association, National Institute for Health and Care Excellence (NICE), and American Society of Hypertension guidelines, recommend self-monitoring for PE symptoms and recording of blood pressure for pregnant women at high risk for $\mathrm{PE} / \mathrm{E}$ (HRPE/E) because of their potential benefits such as effective control of blood pressure, early risk identification, and treatment, and cost savings due to fewer hospital visits. ${ }^{4-6}$ Self-monitoring also has a role in preventing conditions like white coat hypertension and masked hypertension in pregnant women at HRPE/E. WHO suggests home blood pressure monitoring for pregnant women at HRPE/E to detect changes in blood pressure between antenatal visits and to ensure care continuity. ${ }^{7}$

Digital health interventions (DHIs) are increasingly being used to support pregnant women at HRPE/E for remote monitoring of blood pressure and symptoms. To date, four reviews explored the use of digital tools for remote monitoring of pregnant women at HRPE/E. Aquino et al reported 16 unique, feasible and cost-effective telemonitoring interventions to support pregnant women with hypertensive disorder of pregnancy. ${ }^{6}$ However, the review mainly focused on telemonitoring interventions for remote blood pressure monitoring of pregnant women. The review also primarily identified studies from high-income countries like the UK, USA and Belgium. ${ }^{6}$ Lanssens et al reported 14 studies from 1988 to 2010 that used telemonitoring interventions for pregnant women during the prenatal period. ${ }^{8}$ This review, however, used a narrow time range and focused on telemonitoring solutions implemented in high-income countries for pregnant women at high risk for gestational diabetes and preterm labour. In addition, the included studies had a high methodological risk of bias. When only studies with low risk of bias were considered, the added value of telemonitoring became less pronounced. ${ }^{8}$ Rivera-Romero et al captured only 11 studies conducted in high-income countries, on mHealth interventions for the hypertensive disorder of pregnancy. ${ }^{9}$ The included studies showed positive results in the improvement of maternal health and acceptability of solutions, although most of the studies involved a small number of participants, and none were complete clinical studies. ${ }^{9}$ van den Heuvel et al reported 12 studies on the use of telemonitoring and teleconsulting interventions to improve pregnancy care generally. ${ }^{10}$ The review did not focus on the use of eHealth for the hypertensive disorder of pregnancy and generally included all aspects of perinatal care.

These four reviews provided foundational information on the use of telemonitoring to support high-risk pregnant women in antepartum and postpartum period. However, quality evidence on the appropriate use of DHIs to support pregnant women at HRPE/E in LMIC is scarce. None of the reviews extensively documented the use of DHIs in LMICs for the early diagnosis and management of pregnant women at HRPE/E. This gap highlights the need to explore the potential role of DHIs to support pregnant women at HRPE/E in LMICs. This review aims to systematically explore the available literature on the use of DHIs to support early detection and management of PE/E in LMICs.

\section{METHODS}

The 'Preferred Reporting Items for Systematic Reviews and Meta-Analyses extension for Scoping Reviews' (PRISMA-ScR) checklist was used to guide the design and reporting of this scoping review. ${ }^{11}$. The review was registered in the Open Science Framework-Center for Open Science on 19 October 2020 (Registration link: https:// osf.io/gncvj). The review was guided by the methodological framework by Levac et $a l^{2}$ and Arksey et $a l^{13}$ to examine articles describing the use of digital health solutions to support early detection and management of $\mathrm{PE} / \mathrm{E}$ in LMICs.

\section{Identifying research question}

The main research question for this scoping review is: What is known in the literature about DHIs that have been used to support pregnant women at HRPE/E in LMICs?

Our study has used the broad population, concept and context (PCC) framework recommended by the Joanna Briggs Institute for Scoping Reviews. The operationalisation of PCC framework for our scoping review include: population (pregnant women at $\mathrm{HRPE} / \mathrm{E}$ ), concept (DHIs) and context (LMICs).

\section{Eligibility criteria}

The review included studies that involved pregnant women at HRPE/E and implemented the digital health solutions to support early detection and management of PE/E in LMICs. For this scoping review, the DHIs included wearable devices, predictive models operationalised through clinical applications, health information technologies, health management systems, and other innovations related to mobile health, telehealth and telemedicine that can guide diagnosis, monitoring and treatment. ${ }^{14}$ The review included only English-language studies, which were conducted in LMICs. The World Bank's 2020 country classification list was used to select LMICs with a Gross National Income per capita between US $\$ 1036$ and US $\$ 4045 .{ }^{15}$ The review primarily aimed to include original and primary research studies, including experimental studies (eg, randomised controlled trials (RCTs), quasi-experimental studies), observational studies (eg, cohort, case-control, cross-sectional, qualitative studies) and study protocols. All types of reviews, meta-analyses, letters to editors, commentaries, viewpoints, news articles, abstracts and books were excluded. Articles published between 1 January 2000 and 20 October 2020, were included, given that DHIs prior to 2000 would likely have little applicability for current implementation (online supplemental file 1: eligibility criteria).

\section{Information sources and search strategy}

Five main electronic databases were searched including Excerpta Medica Database (EMBASE), Medical Literature 
Analysis and Retrieval System Online (MEDLINE), Cochrane Central Register of Controlled Trials, Cochrane Database of Systematic Reviews, and Cumulated Index to Nursing and Allied Health Literature (CINAHL). A supplementary search was conducted using the first seven pages of Google Scholar to capture peer-reviewed literature on the use of DHIs to support pregnant women at HRPE. The reference lists of relevant systematic reviews and final included articles were also handsearched to find pertinent studies. The search strategy was developed with the assistance of an expert librarian specialising in health services research. It included four main concepts of interest: target population (pregnant women), health condition (PE), intervention (digital health tools) and settings (LMICs). The search strategy included both keywords and subject headings such as $\mathrm{MeSH}$, and Emtree (online supplemental file 2: search strategy).

\section{Selection procedure}

Records from all the electronic databases were exported to Endnote software for screening purposes. The primary reviewer (ASF) developed a predefined screening form, and pilot testing was carried out using 10 randomly selected articles to ensure appropriate screening reliability among the two reviewers (ASF and NA), which was found to be $90 \%$. All articles were independently screened by the two reviewers to exclude those that did not fulfil the inclusion criteria. Two reviewers then met to review any discrepancies which were discussed until a consensus was reached.

The initial search found a total of 4078 articles. After deduplication, 3389 titles and abstracts were screened by the two reviewers (ASF and NA) to evaluate whether they met the eligibility criteria. Of these, 72 records were found to be eligible for full-text screening by the two reviewers. Finally, 19 articles were identified after the full-text screening that met the inclusion criteria for this review. ${ }^{16-34}$ Fifty-three articles were excluded for the following reasons: (1) the study was not reported in the English language; (2) the publication did not talk about pregnant women at HRPE; (3) the research did not include any of the DHIs; (4) the publication was a conference abstract, review, editorial, commentary or (5) the study implemented the DHIs for pregnant women at HRPE in high-income countries. The study selection procedure was recorded according to the PRISMA-ScR flow diagram (figure 1).

\section{Data extraction}

A data abstraction form was designed collectively by the research team to determine appropriate variables such as study characteristics, type of DHIs, intervention description and study outcomes (online supplemental file 3: data abstraction form). To ensure consistency in the data extraction process, the form was pilot tested using three randomly selected articles, which resulted in consistent data being abstracted by both reviewers. Both reviewers (ASF and NA) independently completed the data extraction sheet for each of the 19 final articles. The data abstraction sheets of both the reviewers were compared with confirm that all major results were included in the scoping review. In the case of inconsistencies between the data extraction sheets from the two reviewers, a third reviewer would have been invited to make a final decision, but no inconsistencies were found.

\section{Data analysis}

An inductive approach was used to thematically organise and summarise the results from the included articles to explore our research question. The extracted results from each article were read several times to identify frequent patterns, similarities and differences on the use of DHIs to support pregnant women at HRPE in LMICs. The identified emerging patterns were organised into five thematic groupings including study characteristics, overview and appraisal of included studies, purpose of DHIs, users of DHIs, and types of outcomes examined by the included studies. The first, and last author discussed the results and agreed on the final groupings of the results.

\section{Patient and public involvement}

No patients or members of the public were involved in the protocol design and conduct of the scoping review.

\section{RESULTS}

\section{Study characteristics}

A total of 19 publications describing 7 unique studies were included in this review. The included articles were published between 2009 and 2020. Of these 19 articles, a total of 16 articles described studies that were conducted in South Asia and sub-Saharan Africa, one article described a study conducted in Africa, Southern Asia and the Middle East, and the remaining two articles described studies conducted in unspecified resource-poor settings (LMICs) (online supplemental file 4: overview of the included articles).

The 19 articles were classified into three types of articles: observational studies $(n=12)$, experimental studies $(\mathrm{n}=4$ including two RCTs) and protocol papers $(\mathrm{n}=3)$. All included articles reported the use of DHIs for antepartum women. The articles reported varying eligibility criteria for selecting high-risk pregnant women for different DHIs. Some articles selected high-risk pregnant women based on the NICE guidelines, ${ }^{20}$ specific age groups such as pregnant women aged $15-49$ years, ${ }^{22}$ while a few articles selected pregnant women based on their residential area such as women living in study catchment area, ${ }^{23}$ permanent resident of the particular area, or non-resident who delivered in the study area. ${ }^{18}$ Most DHIs collected blood pressure, heart rate and pulse oximetry, with some innovations collecting data on additional indicators such as demographic data, haemoglobin, urine dipstick test to detect proteinuria and glucose, other urinary markers and PE symptoms. Only one article reported the use of international guideline (NICE clinical guideline 107) to 


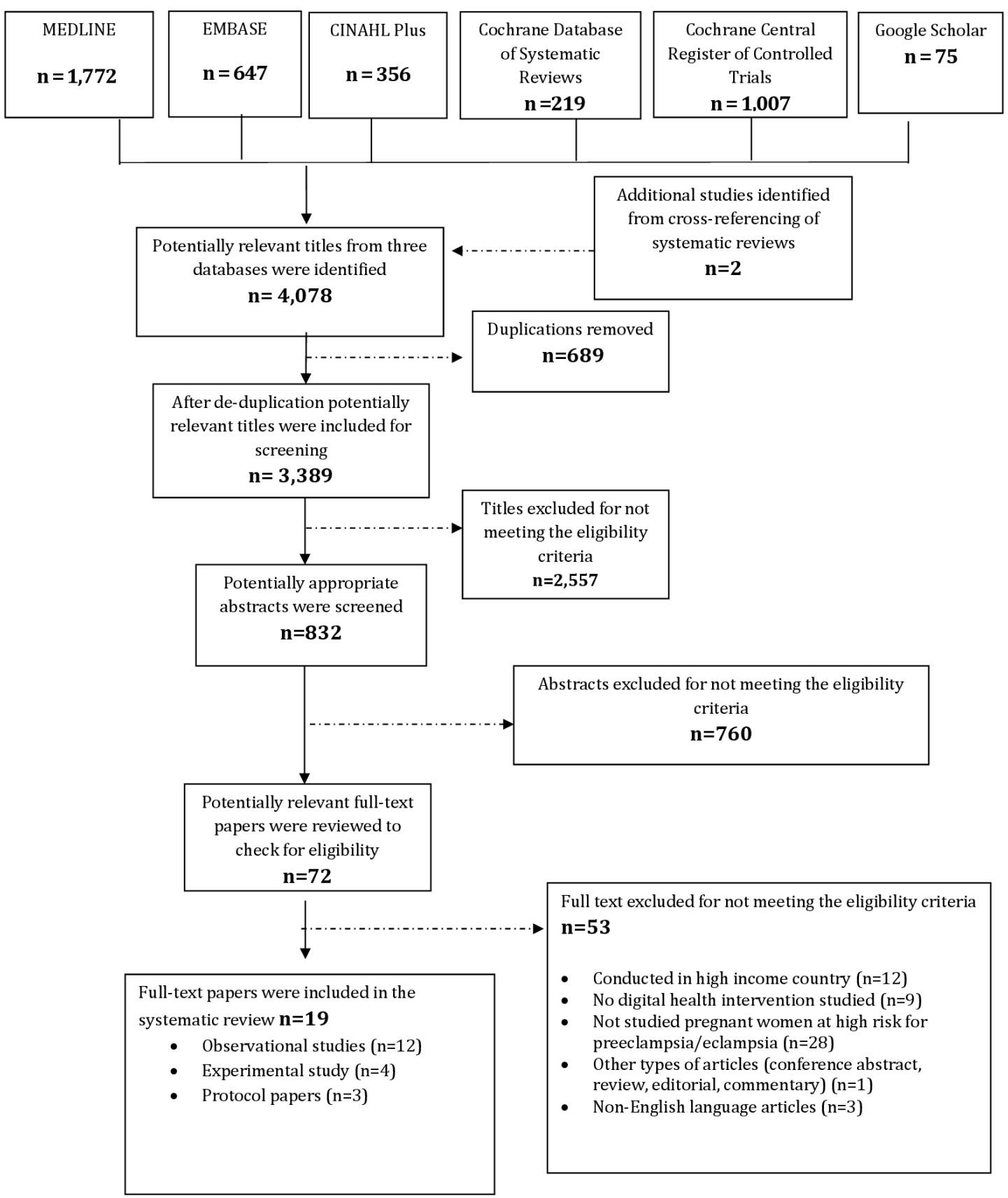

Figure 1 PRISMA-ScR flow diagram for database search of studies. PRISMA-ScR, Preferred Reporting Items for Systematic reviews and Meta-Analyses extension for Scoping Reviews.

determine blood pressure thresholds ${ }^{28}$ (online supplemental file 5: DHIs characteristics).

Seven articles described the application of theoretical frameworks to guide the implementation and evaluation of digital health tools, including the technology acceptance model,${ }^{25}$ diffusion of innovation model, ${ }^{26}{ }^{31}$ three delay model, ${ }^{26}{ }^{29}$ normalisation process theory, ${ }^{23}$ medical research council framework ${ }^{34}$ logic models, ${ }^{31}{ }^{34}$ realist evaluation theories ${ }^{31}$ and cost-effectiveness models. ${ }^{22}$ Two articles described the use of the LambdaNative framework for the development of the 'Pre-eclampsia Integrated Estimate of RiSk (PIERS) on the Move (POTM)' mHealth application. ${ }^{19}{ }^{24}$ The remaining 10 articles did not mention the use of theory or frameworks for the implementation of DHIs.

\section{Overview of the appraisal of included studies}

A total of 10 publications in this review reported research work of the monitoring component of PREEMPT (PE/E Monitoring, Prevention \& Treatment) project by Von Dadelszen et al, University of British Columbia. ${ }^{17-1922-24}$ 29-31 33 The elements of the monitoring component include predictive models, Community Level Interventions for PE (CLIP) and integrated mHealth applications. The PRE-EMPT initiative involved the work of the following research groups: CLIP Pakistan working 
group, CLIP India working group, CLIP trial collaborative group and MiniPIERS and FullPIERS study working group. The PRE-EMPT project was funded through the Bill \& Melinda Gates Foundation (US\$25.9 million).

A total of four articles reported research work of CRADLE vital sign alert (VSA) trial led by Nathan et $a l$, which aimed to evaluate the ability of the device to accurately detect abnormalities in women's vital signs during pregnancy. ${ }^{26-28} 34$ The remaining five publications reported five unique DHIs to support pregnant women at HRPE including the Congo Red Dot test, ${ }^{21}$ a hypothetical telemonitoring programme, ${ }^{20} \mathrm{a}$ new hypertension detector ${ }^{32}$ an integrated diagnostic and clinical decision support system named 'Bliss4Midwives' $(\mathrm{B} 4 \mathrm{M}),{ }^{16}$ and a smart wristwatch (called the F1 smart wristwatch) for blood pressure monitoring of expectant mother. ${ }^{25}$

Following PRISMA-ScR guidelines, each of the abovementioned included article was reviewed to identify emerging themes related to the use of DHIs to support pregnant women at HRPE in LMICs. The key themes that emerged from the observational and experimental studies and protocol papers are as follows: (1) purpose of DHIs including risk prediction and monitoring of highrisk pregnant women; (2) users of DHIs including healthcare providers (HCPs), caregivers and pregnant women; (3) types of outcomes examined in included studies including maternal and neonatal health outcomes, usability and acceptability and intervention feasibility.

\section{Purpose of DHIs}

This review reports nine unique DHIs from 19 included articles to support pregnant women at HRPE/E in LMICs. These unique interventions are clustered into two main groups based on their purpose: predicting risk of adverse maternal health outcomes $(n=2)$ and monitoring highrisk pregnant women to manage $\mathrm{PE} / \mathrm{E}(\mathrm{n}=7)$. Most articles $(n=7)$ described the use of more than one unique DHI (figure 2).

\section{Predicting risk of adverse maternal health outcomes}

Five observational studies and two RCTs described the use of two unique clinical predictive models named fullPIERS $^{19}$ and miniPIERS ${ }^{17-19}{ }^{24} 29-31$ to facilitate the prediction of adverse maternal outcomes occurring as a result of PE based on demographics, symptoms, clinical signs (including $\mathrm{SpO} 2$ ) and laboratory tests. In order to implement these predictive models, the mobile application 'POTM' was developed as an interface to enable healthcare workers to easily determine the risk of adverse maternal health outcomes. One article reported the use of both the miniPIERS and fullPIERS predictive models, ${ }^{19}$ while six articles only reported the use of the miniPIERS model to predict adverse health outcomes among

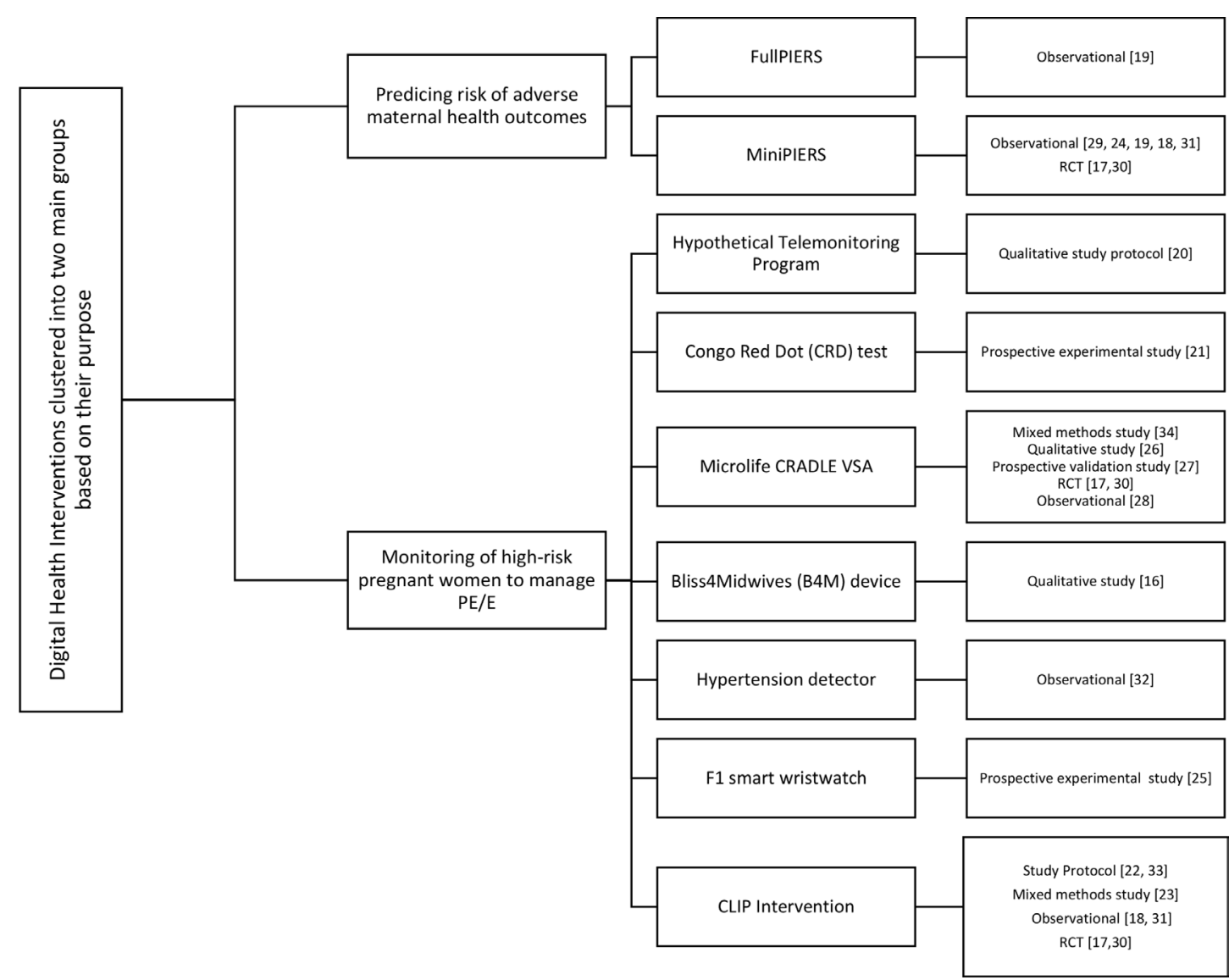

Figure 2 Classification of the included studies based on the purpose of digital health interventions. CLIP, community-level interventions for PE; PE/E, pre-eclampsia/eclampsia; RCTs, randomised controlled trials. 
pregnant women with PE/E in LMICs. ${ }^{17} 1824$ 29-31 Payne et al described the development process of the miniPIERS model to identify pregnant women at HRPE/E in five LMICs using simple-to-measure indicators: personal demographics (gestational age); clinical signs (blood pressure readings and proteinuria); and PE symptoms (headache, visual disturbances, chest pain, dyspnoea, vaginal bleeding and abdominal pain). ${ }^{29}$ The fullPIERS model included additional predictors such as $\mathrm{SpO} 2$ and laboratory tests, to calculate a risk score for pregnant women.

\section{Monitoring high-risk pregnant women for managing PE/E conditions}

The review identified seven unique DHIs for continuous monitoring high-risk pregnant women for managing $\mathrm{PE} / \mathrm{E}$ including one diagnostic test named Congo Red Dot for monitoring misfolded protein in the preeclamptic urine, ${ }^{21}$ CLIP intervention for monitoring blood pressure among high-risk women through community health workers, ${ }^{171822} 23303133$ as well as five unique devices for monitoring blood pressure. ${ }^{16} 202527283234$ The five unique devices for measuring blood pressure among high-risk pregnant women include the Microlife CRADLE VSA device, ${ }^{27} 2834$ the B4M' device, ${ }^{16}$ a new hypertension detector device, ${ }^{32}$ hypothetical telemonitoring programme ${ }^{20}$ and the F1 smart wristwatch. ${ }^{25}$

The Congo Red Dot test was evaluated in a prospective experimental study design. The Congo Red Dot test requires minimal specialised equipment and enables minimally trained personnel to diagnose $\mathrm{PE}$ in resourcelimited healthcare settings. The test was developed in 2016, based on the ability of constituents in pre-eclamptic urine to bind the amyloidophilc dye Congo Red. At the core of the test is the discovery that pre-eclamptic women eliminate misfolded proteins in their urine, a molecular feature that is proportional to disease severity. ${ }^{21}$

The CLIP intervention was implemented in Mozambique, Pakistan, India, and Nigeria as part of cluster RCTs (cRCTs). ${ }^{171822} 233031$ The implementation of CLIP intervention involved scaling-up of existing community health workforce to provide community engagement and community health worker-led app-guided monitoring for high-risk pregnant women for hypertension. Community health workers were able to undertake all aspects of the app-guided visits, and approximately $10 \%$ of pregnant women were found to be hypertensive.

As a first example of blood pressure measurement device, Nathan et al assessed the accuracy of the Microlife 3AS1-2 blood pressure device in 2014 for use in pregnancy and PE in a low-resource setting. ${ }^{27}$ The study recruited a total of 45 pregnant women, of whom 15 had PE, from Kimberley Hospital in South Africa. The study concluded that the device can be recommended for use in pregnancy, including $\mathrm{PE}$ as it fulfils the requirements stipulated by the WHO for an automated blood pressure device suitable for use in antenatal clinics and primary healthcare facilities of LMICs. The device has been extensively validated for accuracy, usability, and acceptability in low-resource settings. ${ }^{27}$ The device calculates the pregnant woman's risk of hypovolaemic or septic shock and alerts frontline healthcare workers about vital sign abnormalities through a traffic light early warning system display. In 2018, a 3-month mixed-methodology feasibility study was conducted to incorporate the CRADLE VSA device into routine maternity care in 10 low-income sites. ${ }^{34}$ Primary, secondary and tertiary facilities were allocated devices and training packages consisting of a shortanimated film, interactive sessions, booklet, and posters.

As a second example, a study conducted in Ghana used the B4M device which included infrared sensors to measure haemoglobin, a self-inflating cuff for blood pressure measurement, and an automated reader for urinary protein and glucose through dipsticks. The device facilitated non-invasive screening of PE and served as an integrated diagnostic and clinical decision support device for PE. ${ }^{16}$ The third example of a device for blood pressure monitoring was a new hypertension detector, developed by Thakor et al, which was compared in an observational study with other traditional devices for use in developing countries to support pregnant women at HRPE/E. ${ }^{32}$ The new device was found to be more accurate and easy-to-use than CRADLE VSA and other devices, due to the reduced number of steps required for use. ${ }^{32}$ As a fourth example of a device for blood pressure monitoring was a hypothetical telemonitoring programme, ${ }^{20}$ which was described in a qualitative study protocol. The study intended to explore the perspectives, needs, and preferences of a telemonitoring programme for pregnant women at HRPE in a tertiary health facility of Karachi, to inform future implementation.

Finally, one prospective experimental study used a wearable device called the F1 smart wristwatch that included an integrated chip for sensing blood pressure readings and displaying real-time data on the screen. The smartwatch on the expectant mother's wrist takes blood pressure readings and transfers them by Bluetooth to their phone at regular intervals to facilitate personal health tracking. The caregiver can access the expectant mother's records, as well as receive alerts on blood pressure readings. $^{25}$

Both of these purposes used mobile phone applications as an interface to facilitate data collection, decision making and communication between health workers and pregnant women. The majority of these studies used the POTM application ${ }^{17-24303133}$ to facilitate the collection of relevant clinical data during antenatal visits. The application was used by community health workers in India, Pakistan, Nigeria, and Mozambique, as part of a CLIP cluster RCT. ${ }^{17} 30$ The POTM platform combined two interventions, which were the miniPIERS model and a Phone Oximeter to accurately predict the risk score for pregnant women at HRPE/E in LMICs. The application generated a risk estimate which enabled community health workers and other HCPs to stratify high-risk pregnant women, escalate care and make referrals to 
the facility. In addition, Jonas et $a l$ s study used a mobile application for administrating CRD test for monitoring misfolded protein in the pre-eclamptic urine. ${ }^{21}$ Finally, the Feroz et als study protocol described a hypothetical mobile-based telemonitoring programme which would serve as a communication aid between nurses and highrisk pregnant women. ${ }^{20}$

\section{Users of DHls}

Most articles involved HCPs $(n=17)$ as the targeted primary users of the DHIs, while only two articles had pregnant women and caregivers as the primary users of the DHI. ${ }^{20}{ }^{25}$ The articles described various healthcare workers as the users of the DHIs, including mid-level HCPs, community-based HCPs, female health supervisors, semi-literate volunteers, community health nurses, female health workers, midwives and accredited social health activists. Sixteen articles included information on the training of patients and HCPs on how to use the DHI, interpret physiological metrics, and take actionable measures for critical results. ${ }^{16} 1819$ 21-24 26-34 The HCPs received advanced training to enhance their assessment skills and ability to facilitate the overall management of pregnant women at HRPE/E. Three articles did not specify the training component for either HCPs or patients. ${ }^{172025}$

\section{Type of outcomes examined}

The included articles $(n=19)$ reported on three major outcomes: (1) maternal and neonatal health outcomes $(\mathrm{n}=4),(2)$ usability and acceptability $(\mathrm{n}=5)$ and (3) intervention feasibility $(n=7)$ (online supplemental file 6 : outcomes of DHIs).

\section{Maternal and neonatal health outcomes}

Four articles examining maternal and neonatal health outcomes were observational studies $(n=2)$ and RCTs $(\mathrm{n}=2) .{ }^{17} 18 \quad 28 \quad 30$ Maternal health outcomes included magnesium sulfate use, hospital admissions, critical care unit (CCU) admissions, birth preparedness, complication readiness, facility delivery attended by skilled birth attendants, and adverse maternal outcomes such as an increase in kidney injury, maternal morbidity, and mortality. For example, Nathan et al's observational study evaluated the association between blood pressure measurements and adverse outcomes in women with PE using CRADLE VSA traffic light early warning system. The study demonstrated that the risk of maternal death, eclampsia and perinatal death was similar across the women who triggered a yellow or red light on the CRADLE VSA. However, the risk of kidney injury, maternal use of magnesium sulfate, maternal CCU admission and preterm delivery, was greater for those who triggered a red light, compared with a yellow light. ${ }^{28}$ The two RCTs reported nonsignificant findings regarding maternal morbidity and mortality for participants in the DHI arm. ${ }^{17}{ }^{30}$ Neonatal health outcomes included stillbirths, fetal and neonatal morbidity, and mortality. Only one of the two RCTs reported a reduction in stillbirths $(0.89$ (95\% CI 0.81 to $0.99) ; \mathrm{p}=0.03$ ) in the DHI group; however, no impact on neonatal morbidity or mortality was reported for participants in the DHI group. ${ }^{30}$

\section{Usability and acceptability}

Five articles reported on the usability and acceptability of DHIs in LMICs. ${ }^{19}$ 24-26 32 The articles mentioned pregnant women, caregivers and HCPs' experience of use of DHIs in LMICs. Usability outcomes included: trust in technology, ease of use, content richness, perceived usefulness and user satisfaction. For instance, Musyoka $e t$ $a l$ s study found that a 24-hour ambulatory blood pressure monitoring system has a great potential for actual adoption in healthcare systems in low-income and middleincome countries, given its simplicity and affordability. ${ }^{25}$ The study found that content richness had a slightly positive linear effect on perceived ease of use, while there is a slightly negative relationship between content richness and perceived usefulness. ${ }^{25}$ Lim et al used the computer systems usability questionnaire to assess the usability of the POTM mHealth application. ${ }^{24}$ Nurses and midwives who participated in the study rated the usability high for the integration of these technologies and thought it would help their fieldwork. The study found that usability issues were often related to navigation of the app and phone features such as scroll wheels, touch screen use, etc. In a study by Nathan et al, most HCWs perceived the CRADLE device to be easy to use; however, some described manual inflation as tiring, particularly when measuring vital signs in obese and hypertensive women. ${ }^{26}$ Dunsmuir $e t a l$ s study reported on the usability of CLIP POTM application; the CLIP trial received requests from different countries for modifications in POTM to consider different user needs and cultural differences leading to modified application versions for each country. ${ }^{19}$

\section{Intervention feasibility and fidelity}

Most articles $(n=7)$ reported on the feasibility and fidelity of DHIs for pregnant women at HRPE/E in LMICs in order to provide evidence on the evaluation of DHIs for replication and scale-up of successful DHIs. ${ }^{16212327293134}$ Study outcomes included: fidelity and accuracy of the CRADLE VSA device, MiniPIERS model development and validation, understanding of enabling and impeding factors for CLIP trial implementation, experiences of pregnant women with B4M intervention and cost-effectiveness of the Congo Red Dot test. For example, Payne et als study informed that miniPIERS model has a reasonable ability to identify women at increased risk of adverse maternal outcomes associated with the hypertensive disorders of pregnancy. ${ }^{29}$ Nathan $e t a l$ s another study assessed the accuracy of Microlife 3AS1-2 blood pressure device for accuracy for use in pregnancy in LMICs. The authors concluded that the device can be recommended for use in pregnancy, including PE as it meets the standards stipulated by the WHO for automated blood pressure devices suitable for low-resource settings. ${ }^{27}$ One mixed-methods 
study reported high fidelity of the implementation of the CRADLE VSA device, with improved HCPs ability to make clinical decisions, escalate care, and make immediate referrals in case of emergency. ${ }^{34}$ The study by Khowaja et al reported factors associated with the feasibility of the CLIP trial implementation including community mobilisation, institutional support, system integration, knowledge gaps, lack of trained personnel, cultural myths and misconceptions, poor health service quality and high cost of care. ${ }^{23}$

\section{DISCUSSION}

\section{Principal findings}

This review summarises evidence on the existing DHIs to support pregnant women at HRPE/E in LMICs. Given that most articles (11 out of 19) were published between 2015 and 2020, the novelty of DHIs use to support pregnant women with HRPE/E was indicated. Only nine unique DHIs were identified in this review from 19 included articles, reflecting the limited understanding and use of DHIs to support pregnant women in LMICs. Most included articles used observational and exploratory research methods to study DHIs. This suggested the need for concerted efforts to learn from small innovation projects and deployments as outlined in WHO guide on monitoring and evaluation of DHIs. ${ }^{35}$ Most articles in this review did not report information on the blood pressure thresholds, which limited our understanding of standardised blood pressure thresholds used in LMICs. The explicit reporting of standardised blood pressure thresholds could help in designing effective clinical decision support systems for monitoring pregnant women in LMICs. ${ }^{36}$

\section{Implementation barriers and strategies for DHIs}

The Microlife CRADLE VSA blood pressure monitoring device has been extensively validated for use in LMICs for pregnant women. ${ }^{27} 28$ However, HCPs faced several barriers during the implementation of CRADLE VSA device including lack of supportive supervision for device use, high staff turnover and poor availability of the device, poor battery life of device, misleading displays, broken hand pump, tubing and broken charging ports. ${ }^{34}$ Nathan $e t$ al and Vousden et al suggested a range of implementation strategies to address known barriers, prior to scale-up, including recognising designated device champions who can provide in-depth local training and support for device use, emphasising the importance of a device training package (short animated film, interactive sessions, booklet and posters), updating training materials to explain the traffic light alert system, providing chargers in addition to the USB cable, and ensuring an adequate supply of VSA devices. ${ }^{28}{ }^{34} \mathrm{Lim}$ et al's study mentioned that the general unfamiliarness of using touch screen smart phones was reported as the major barrier faced during the implementation of POTM application. ${ }^{24}$ Abejirinde $e t$ $a l$ 's study trained users on the technical and operational functions of the device to address technical and procedural issues including software freezes, slow response time and low user dexterity with operating the device. ${ }^{16}$

\section{Research gaps and suggestions for future research}

Enabling the use of DHls by pregnant women as end-users instead of HCPs as end-users

Most articles in this review targeted DHIs at HCPs who have less formal training and education, as opposed to studies conducted in high-income countries where DHIs have been targeted at family physicians and clinicians who have specialised medical training. ${ }^{6}$ This review identified only one study that targeted DHI at pregnant women for personal health tracking ${ }^{25}$; however, DHIs implemented in high-income countries are often targeted for use by pregnant women to improve maternal health behaviours and maternal-fetal health outcomes. ${ }^{37}$ Given the increasing cell phone penetration in LMICs, ${ }^{38}$ there is an opportunity to use mobile phone technology to target DHIs at the patient level (pregnant women) to encourage personal health tracking. Yet, health informatics researchers should consider issues of technological literacy, user characteristics (age, gender, computer skills, experience), cultural factors and socioeconomic status when designing and implementing DHIs in the LMIC context. ${ }^{39}$ None of the studies delivered targeted client instructions via a digital platform, in response to abnormal blood pressure readings or signs and symptoms of PE. In high-income countries, some digital health platforms have delivered manual or automated targeted instructions to the pregnant women to provide information about medications, referrals and diet. ${ }^{40}$ LMICs can learn from the experiences of high-income countries for developing context-specific digital platforms that can facilitate targeted client communication between providers and pregnant women. Evidence suggests that the targeted client communication for transmission of health information, health event alerts and reminders, and diagnostic results have shown positive impacts on health behaviours and health outcomes in high-income countries. ${ }^{41}$

\section{Using multidisciplinary team approach for designing DHIs}

None of the DHIs used a multidisciplinary team approach for monitoring of pregnant women for PE/E. Blandford et al suggest that DHIs should involve collaboration between different cadres of HCPs across all levels of the health system, to achieve the full potential of digital intervention. ${ }^{42}$ For instance, a nurse or midwife at a primary level could communicate about a pregnant women's health condition to a clinician at a secondary institution to seek recommendations for managing pregnant women at HRPE/E. Murray et al suggest that high-quality research in the digital health field requires fertile multidisciplinary collaborations that draw on insights and experience from multiple fields, including clinical medicine, health services research, behavioural science, education, engineering and computer science. ${ }^{43}$ Thus, research 
aimed at designing and evaluating DHIs to support pregnant women at HRPE/E should draw insights from collaborators belonging to diverse disciplines including obstetricians and gynaecologists, telemedicine experts, knowledge users, HCPs (nurses, doctors), public health specialists, maternal health specialists, health services researchers, as well as patient partners.

\section{Exploring telemedicine use to enable remote consultation between pregnant women and HCPs}

Most articles used DHIs for the prediction of adverse maternal outcomes, data collection and decision aid, diagnostic and clinical decision support, and personal health tracking. There is a lack of evidence on using DHIs for referral coordination, teleconsultation between pregnant women and HCPs, communication between the HCP and their supervisor, and HCPs' training. Telemedicine has been extensively used in high-income countries for providing a range of obstetrical services such as using videoconference to replace in-person visits, implementing at-home monitoring, enabling consultation with remote specialists, earlier postpartum follow-up visits and access to lactation consultants. ${ }^{44}$ This evidence shows the potential of using telemedicine for pregnant women at HRPE/E in LMICs to enable remote monitoring and remote consultation between pregnant women and providers.

\section{Monitoring and evaluating the implementation and effectiveness} of DHIs

Most articles reported on intervention feasibility, usability and acceptability outcomes. Two RCTs reported nonsignificant findings for maternal morbidity, mortality and neonatal deaths ${ }^{17} 30$ with only one RCT that reported a significant difference in stillbirth rate in DHIs group. ${ }^{30}$ This suggests the need of conducting more experimental studies such as RCTs to evaluate the efficacy and effectiveness of diverse DHIs to improve maternal and child health outcomes. In the review, only one study protocol described the methodology to conduct an economic evaluation of the CLIP package in South Asian and African countries. ${ }^{22}$ This shows the paucity of evidence on the economic impact of DHIs to support pregnant women with $\mathrm{PE} / \mathrm{E}$. Ramsey et al recommend that future clinical trials should incorporate cost-analysis of DHIs as there is mounting evidence on embedding economic evaluations within clinical trials to build a robust cost-effectiveness model that has high internal validity and timeliness. ${ }^{45}$ The articles included in this review did not extensively identify facilitators and challenges encountered during the implementation of DHIs for pregnant women with $\mathrm{PE} / \mathrm{E}$ in LMICs, unlike many studies conducted in highincome countries. ${ }^{6}$ This review identified only a few facilitators: easy to use technology, trust in technology, and availability of diagnostic service at the point of care. This indicates the need to examine and report on enablers and barriers faced when employing DHIs for pregnant women at HRPE/E across the stages of design, development, implementation and evaluation.

In summary, this scoping review suggests four recommendations for future research: (1) enable the use of DHIs by pregnant women as end-users to encourage personal health tracking including individualised patient instructions; (2) consider a multidisciplinary team approach when designing DHIs for pregnant women at HRPE/E; (3) explore the potential of using telemedicine in LMICs to enable remote consultation between pregnant women and health providers; (4) conduct further studies including prospective longitudinal and experimental studies to establish the implementation effectiveness and efficacy of DHIs to support pregnant women at HRPE; exploratory studies to identify barriers and enablers associated with the development, implementation and evaluation of DHIs; and economic evaluations of DHIs within large clinical trials to identify cost-effective DHIs.

\section{CONCLUSION}

The current evidence base is sparse but shows some potential for the use of different DHIs to support pregnant women in early diagnosis of $\mathrm{PE} / \mathrm{E}$ through predicting the risk for adverse maternal health outcomes and monitoring high-risk pregnant women for $\mathrm{PE} / \mathrm{E}$ through devices and other DHIs. Limited evidence exists on types, benefits, cost-effectiveness and outcomes of DHIs. The weak evidence may impede the adoption of these promising technologies in community and healthcare settings to support pregnant women at HRPE/E in LMICs. Future research work should target DHIs at the pregnant women level to promote personal health tracking with targeted instructions for pregnant women, consider a multidisciplinary team approach for designing DHIs, explore the role of telemedicine to enable remote consultation between pregnant women and HCPs, and evaluate the implementation and effectiveness of DHIs.

Contributors ASF and ES authors conceptualised and designed the study. ASF screened the articles and performed data extraction, synthesised the data and drafted the manuscript. NA independently performed screening of articles. ES served as the senior author including participating in the data analysis and providing critical feedback on the manuscript. All authors read and approved the final manuscript. ASF accepts responsibility for this work and is the guarantor.

Funding The authors have not declared a specific grant for this research from any funding agency in the public, commercial or not-for-profit sectors.

Competing interests None declared.

Patient consent for publication Not applicable.

Ethics approval This study does not involve human participants.

Provenance and peer review Not commissioned; externally peer reviewed.

Data availability statement No data are available. No additional data are available.

Supplemental material This content has been supplied by the author(s). It has not been vetted by BMJ Publishing Group Limited (BMJ) and may not have been peer-reviewed. Any opinions or recommendations discussed are solely those of the author(s) and are not endorsed by BMJ. BMJ disclaims all liability and responsibility arising from any reliance placed on the content. Where the content 
includes any translated material, BMJ does not warrant the accuracy and reliability of the translations (including but not limited to local regulations, clinical guidelines, terminology, drug names and drug dosages), and is not responsible for any error and/or omissions arising from translation and adaptation or otherwise.

Open access This is an open access article distributed in accordance with the Creative Commons Attribution Non Commercial (CC BY-NC 4.0) license, which permits others to distribute, remix, adapt, build upon this work non-commercially, and license their derivative works on different terms, provided the original work is properly cited, appropriate credit is given, any changes made indicated, and the use is non-commercial. See: http://creativecommons.org/licenses/by-nc/4.0/.

\section{ORCID iDs}

Anam Shahil Feroz http://orcid.org/0000-0003-0180-0213

Noreen Afzal http://orcid.org/0000-0003-3322-4599

\section{REFERENCES}

1 Goldenberg RL, Jones B, Griffin JB, et al. Reducing maternal mortality from preeclampsia and eclampsia in low-resource countries--what should work? Acta Obstet Gynecol Scand 2015;94:148-55.

2 Khowaja AR, Qureshi RN, Sheikh S, et al. Community's perceptions of pre-eclampsia and eclampsia in Sindh Pakistan: a qualitative study. Reprod Health 2016;13:39-44.

3 Howden-Chapman P. Sdg 3: ensure healthy lives and promote wellbeing for all at all ages, 2017: 81-126.

4 van den Heuvel JFM, Teunis CJ, Franx A, et al. Home-based telemonitoring versus hospital admission in high risk pregnancies: a qualitative study on women's experiences. BMC Pregnancy Childbirth 2020;20:1-9.

5 van den Heuvel JFM, Kariman SS, van Solinge WW, et al. SAFE@ HOME - Feasibility study of a telemonitoring platform combining blood pressure and preeclampsia symptoms in pregnancy care. Eur J Obstet Gynecol Reprod Biol 2019;240:226-31.

6 Aquino M, Munce S, Griffith J, et al. Exploring the use of Telemonitoring for patients at high risk for hypertensive disorders of pregnancy in the antepartum and postpartum periods: Scoping review. JMIR Mhealth Uhealth 2020;8:e15095.

7 WHO. WHO recommendations on antenatal care for a positive pregnancy experience. Geneva, Switzerland, 2016.

8 Lanssens D, Vandenberk T, Thijs IM, et al. Effectiveness of telemonitoring in obstetrics: Scoping review. J Med Internet Res 2017;19:e327.

9 Rivera-Romero O, Olmo A, Muñoz R, et al. Mobile health solutions for hypertensive disorders in pregnancy: Scoping literature review. JMIR Mhealth Uhealth 2018;6:e130.

10 van den Heuvel JF, Groenhof TK, Veerbeek JH, et al. eHealth as the next-generation perinatal care: an overview of the literature. J Med Internet Res 2018;20:e202.

11 de Oliveira BGRB. Preferred reporting items for systematic reviews and meta-analyses extension for scoping reviews (PRISMA-ScR) checklist, 2015.

12 Levac D, Colquhoun H, O'Brien KK. Scoping studies: advancing the methodology. Implement Sci 2010;5:69.

13 O'Malley LJIJSRM. Scoping studies: towards a methodological framework AU-Arksey, Hilary. Int J Soc Res Methodol 2005;8:19-32.

14 Guo C, Ashrafian H, Ghafur S, et al. Challenges for the evaluation of digital health solutions - A call for innovative evidence generation approaches. NPJ Digit Med 2020;3:1-14.

15 Serajuddin U, Hamadeh N. New world bank country classifications by income level: 2020-2021. World Bank Blogs 2020.

16 Abejirinde I-OO, Douwes R, Bardají A, et al. Pregnant women's experiences with an integrated diagnostic and decision support device for antenatal care in Ghana. BMC Pregnancy Childbirth 2018:18:209.

17 Bellad MB, Goudar SS, Mallapur AA, et al. Community level interventions for pre-eclampsia (clip) in India: a cluster randomised controlled trial. Pregnancy Hypertens 2020;21:166-75.

18 Bellad MB, Vidler M, Honnungar NV, et al. Maternal and newborn health in Karnataka state, India: the community level interventions for pre-eclampsia (CLIP) Trial's baseline study results. PLoS One 2017;12:e0166623.

19 Dunsmuir DT, Payne BA, Cloete G, et al. Development of mHealth applications for pre-eclampsia triage. IEEE J Biomed Health Inform 2014;18:1857-64.

20 Feroz A, Saleem S, Seto E. Exploring perspectives, preferences and needs of a telemonitoring program for women at high risk for preeclampsia in a tertiary health facility of karachi: a qualitative study protocol. Reprod Health 2020;17:135

21 Jonas SM, Deserno TM, Buhimschi CS, et al. Smartphone-Based diagnostic for preeclampsia: an mHealth solution for administering the Congo red dot (CRD) test in settings with limited resources. J Am Med Inform Assoc 2016;23:166-73.

22 Khowaja AR, Mitton C, Bryan S, et al. Economic evaluation of community level interventions for pre-eclampsia (clip) in South Asian and African countries: a study protocol. Implement Sci 2015;10:76.

23 Khowaja AR, Qureshi RN, Sawchuck D, et al. The feasibility of community level interventions for pre-eclampsia in South Asia and sub-Saharan Africa: a mixed-methods design. Reprod Health 2016;13 Suppl 1:56.

24 Lim J, Cloete G, Dunsmuir DT, et al. Usability and feasibility of PIERS on the move: an mHealth APP for pre-eclampsia triage. JMIR Mhealth Uhealth 2015;3:e37.

25 Musyoka FM, Thiga MM, Muketha GM. A 24-hour ambulatory blood pressure monitoring system for preeclampsia management in antenatal care. Inform Med Unlocked 2019;16:100199.

26 Nathan $\mathrm{HL}$, Boene $\mathrm{H}$, Munguambe $\mathrm{K}$, et al. The cradle vital signs alert: qualitative evaluation of a novel device designed for use in pregnancy by healthcare workers in low-resource settings. Reprod Health 2018;15:5.

27 Nathan HL, de Greeff A, Hezelgrave NL, et al. An accurate semiautomated oscillometric blood pressure device for use in pregnancy (including pre-eclampsia) in a low-income and middleincome country population: the Microlife 3AS1-2. Blood Press Monit 2015:20:52-5.

28 Nathan HL, Seed PT, Hezelgrave NL, et al. Early warning system hypertension thresholds to predict adverse outcomes in preeclampsia: a prospective cohort study. Pregnancy Hypertens 2018;12:183-8.

29 Payne BA, Hutcheon JA, Ansermino JM, et al. A risk prediction model for the assessment and triage of women with hypertensive disorders of pregnancy in low-resourced settings: the miniPIERS (pre-eclampsia integrated estimate of risk) multi-country prospective cohort study. PLoS Med 2014;11:e1001589.

30 Qureshi RN, Sheikh S, Hoodbhoy Z, et al. Community-Leve interventions for pre-eclampsia (clip) in Pakistan: a cluster randomised controlled trial. Pregnancy Hypertens 2020;22:109-18.

31 Sharma S, Adetoro OO, Vidler M, et al. A process evaluation plan for assessing a complex community-based maternal health intervention in Ogun state, Nigeria. BMC Health Serv Res 2017;17:238.

32 Thakor N, Trachtenberg M, Lee S. Hypertension detector for developing countries. Department of Biomedical Engineering, Johns Hopkins University, 2010.

33 von Dadelszen P, Flint-O'Kane M, Poston L, et al. The precise (pregnancy care integrating translational science, everywhere) network's first protocol: deep phenotyping in three sub-Saharan African countries. Reprod Health 2020;17:51.

34 Vousden N, Lawley E, Nathan HL, et al. Evaluation of a novel vita sign device to reduce maternal mortality and morbidity in lowresource settings: a mixed method feasibility study for the CRADLE-3 trial. BMC Pregnancy Childbirth 2018;18:115.

35 World Health Organization. Monitoring and evaluating digital health interventions: a practical guide to conducting research and assessment, 2016.

36 Nee O. Clinical decision support with guidelines and Bayesian networks, 2010: 117-37.

37 Hussain T, Smith P, Yee LM. Mobile Phone-Based behavioral interventions in pregnancy to promote maternal and fetal health in high-income countries: systematic review. JMIR Mhealth Uhealth 2020;8:e15111.

38 Muir S. To what extent is mHealth an effective intervention in LMICs? 2019.

39 Feroz A, Kadir MM, Saleem S. Health systems readiness for adopting mHealth interventions for addressing non-communicable diseases in low- and middle-income countries: a current debate. Glob Health Action 2018;11:1496887.

40 Haddad SM, Souza RT, Cecatti JG. Mobile technology in health (mHealth) and antenatal care-Searching for apps and available solutions: a systematic review. Int J Med Inform 2019;127: :1-8.

41 WHO. WHO guideline: recommendations on digital interventions for health system strengthening: web supplement 2: summary of findings and grade tables. World Health Organization, 2019.

42 Blandford A, Gibbs J, Newhouse N, et al. Seven lessons for interdisciplinary research on interactive digital health interventions. Digit Health 2018;4:205520761877032.

43 Murray E, Hekler EB, Andersson G, et al. Evaluating digital health interventions: key questions and approaches. Am J Prev Med 2016;51:843-51. 
44 Weigel G, Frederiksen B, Ranji U. Telemedicine and pregnancy care. The Henry J Kaiser Family Foundation, 2020.

45 Ramsey S, Willke R, Briggs A, et al. Good research practices for cost-effectiveness analysis alongside clinical trials: the ISPOR
RCT-CEA Task force report. Value Health 2005;8:10.1111/j.1524-4733.2005.00045.x:521-33. 